\title{
Rapid Continuum Variability in NGC 4151
}

\author{
Rick Edelson \\ Department of Physics and Astronomy, 203 Van Allen Hall, University \\ of Iowa, lowa City, IA 52940, USA
}

\section{Data}

The bright Seyfert 1 galaxy $\mathrm{NGC} 4151$ was monitored intensively by $I U E$, ROSAT, ASCA, CGRO, and ground-based telescopes for 10 days in 1993 December. The source, which was near its peak historical brightness, showed strong, correlated variability at X-ray, ultraviolet, and optical wavelengths (see Fig. 1a). The strongest variations were seen in medium energy $(\sim 1.5 \mathrm{keV}) \mathrm{X}$-rays, with a normalized variability amplitude (NVA) of $24 \%$. Weaker (NVA $=6 \%$ ) variations, uncorrelated with those at lower energies, were seen at soft $\gamma$-ray energies of $\sim 100 \mathrm{keV}$. No significant variability was seen in softer $(0.1-1 \mathrm{keV}) \mathrm{X}$-ray bands. In the ultraviolet/optical regime, the NVA decreased within increasing wavelength, from $9 \%$ to $1 \%$ between $1275 \AA$ and $6900 \AA$.

The phase differences between variations in different bands were consistent with zero lag, with upper limits of $\lesssim 0.15$ day between $1275 \AA$ and the other ultraviolet bands, $\lesssim 0.3$ day between $1275 \AA$ and $1.5 \mathrm{keV}$, and $\lesssim 1$ day between $1275 \AA$ and $5125 \AA$. These tight limits represent more than an order of magnitude improvement over those determined in previous multi-waveband AGN monitoring campaigns. The ultraviolet fluctuation power spectra showed no evidence for periodicity, but were instead well-fitted with a very steep, red power-law.

A detailed version of this contribution will appear as a series of papers on the ultraviolet (Crenshaw et al. 1996), optical (Kaspi et al. 1996) and highenergy data (Warwick et al. 1996), and multi-wavelength analysis (Edelson et al. 1996) in The Astrophysical Journal.

\section{Interpretation}

If photons emitted at a 'primary' waveband are absorbed by nearby material and 'reprocessed' to produce emission at a secondary waveband, causality arguments require that variations in the secondary band follow those in the primary band. The tight interband correlation and limits on the ultraviolet and medium energy $\mathrm{X}$-ray lags indicate that the reprocessing region is smaller than $\sim 0.15$ lt-day in size. After correcting for strong (factor of $\gtrsim 15$ ) line-of-sight absorption (see Fig. $1 b)$, the medium-energy $X$-ray luminosity variations appear adequate to drive the ultraviolet/optical variations. However, the medium energy X-ray NVA is 2-4 times that in the ultraviolet, and the single-epoch, absorption-corrected Xray/ $\gamma$-ray luminosity is only about $1 / 3$ that of the ultraviolet/optical/infrared, suggesting that at most $\sim 1 / 3$ of the total low-energy flux could be reprocessed high-energy emission. 

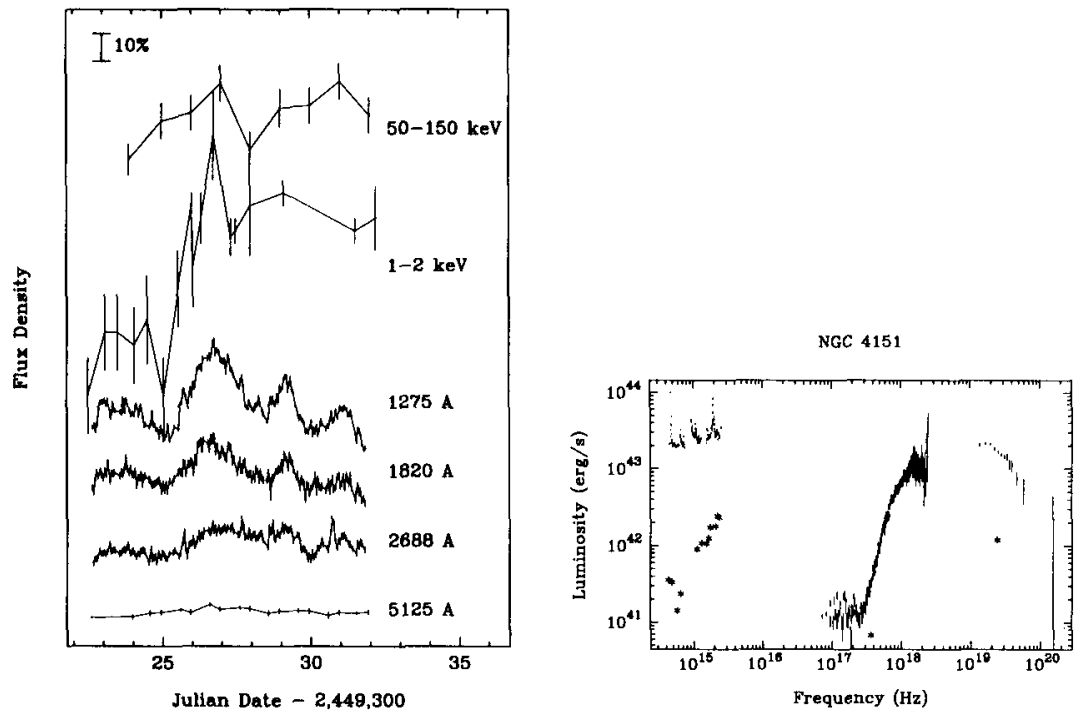

Figure 1. Left: Continuum light curves during the intensive monitoring of NGC 4151, for wavebands centered on $100 \mathrm{keV}, 1.5 \mathrm{keV}, 1275 \AA$, $1820 \AA, 2688 \AA$, and $5125 \AA$. The light curves are on a common scale, but shifted in flux to present them in one figure, so a $10 \%$ flux change is shown. Right: Spectral energy distribution of NGC 4151. Plotted quantities are monochromatic luminosity $\left(4 \pi D^{2} \nu S_{\nu}\right)$ as a function of frequency. The asterisks show the NVA in each of the observing bands, multiplied by monochromatic luminosity.

The strong wavelength dependence of the ultraviolet NVAs is consistent with an origin in an accretion disk, with the variable emission coming from the hotter inner regions and non-variable emission from the cooler outer regions. These data, when combined with the results of disk fits, indicate a boundary between these regions near a radius of order $R \approx 0.07 \mathrm{lt}$-day. No interband lag would be expected as reprocessing (and thus propagation between regions) need not occur, and the orbital time scale of $\sim 1$ day is consistent with the observed variability time scale. However, such a model does not immediately explain the good correlation between ultraviolet and X-ray variations.

\section{References}

Crenshaw, D. M. et al. 1996, ApJ, 470, in press.

Edelson, R. et al. 1996, ApJ, 470, in press.

Kaspi, S. et al. 1996, ApJ, 470, in press.

Warwick, R. et al. 1996, ApJ, 470, in press. 